\section{P 054 THE IMPACT OF BEING PART OF A THET LINKS PARTNERSHIP TO DEVELOP A PALLIATIVE CARE DEGREE PROGRAM IN SUB SAHARAN AFRICA}

Barbara Jack, ${ }^{1}$ Jennifer Kirton, ${ }^{1}$ Karen Frame ${ }^{3} .{ }^{1}$ Edge Hill University, Ormskirk, UK; ${ }^{2}$ Edge Hill University, Ormskirk, UK; ${ }^{3}$ Imperial College Healthcare NHS Trust

\subsection{6/bmjspcare-2014-000654.95}

Background In Sub-Saharan Africa the need for palliative care provision is escalating with rising cancer rates and noncommunicable disease burden. The shortage of trained health care professionals has highlighted the need for increasing the palliative care workforce. A THET Links Health Partnership was established between a UK Faculty comprising clinical and academic palliative care staff from across the UK and Hospice Africa Uganda, to support the development of a palliative care degree in Uganda. UK Faculty supported the Ugandan Faculty in the development and delivery of the degree programme.

Aim To identify the impact on the UK Faculty of participating in the partnership.

Methods A pragmatic evaluation methodology was adopted for the study and data were collected in November 2012, using a confidential descriptive electronic survey, comprising open ended and closed questions. A total population sample approach was used and all 17 UK Faculty invited to participate. Data were analysed using thematic analysis and descriptive statistics for the closed questions. The project was reviewed by West London Research Ethics Committee and considered service evaluation.

Result $82 \%(\mathrm{n}=14)$ UK Faculty participated, and no negative points were raised by any respondent. Several themes emerged from the data including the impact on their UK clinical practice. This included examples of improving their team working and leadership and enhanced diagnostic skills with reduced reliance on technology. The improvement in their teaching skills was widely reported. The personal impact of the experience was also noted including providing personal growth, influencing lifestyle choices and changing their perspectives on life.

Conclusion the impact of being part of this volunteer programme has shown multiple benefits for the participants, not only relating to their clinical work in the UK, but also the personal impacts on their lives. As well as the overarching goal of improved access to palliative care. 